\title{
LA CONSTRUCCION PREFABRICADA DE PASOS INFERIORES AL FERROCARRIL Y SU PUESTA EN OBRA MEDIANTE LA TECNICA DE EMPUJE O DEL CAJON HINCADO
}

\author{
(CONSTRUCTION OF PREFABRICATED RAILWAY UNDERPASSES AND EXECUTION OF THE \\ SAME USING THE THRUST OR DRIVEN-IN CASSION TECHNIQUE)
}

Ramón Escribano Méndez, Ingeniero de Caminos. Gerente de Pasos a Nivel de RENFE

Rafael López Palomar, Ingeniero de Caminos. Jefe del Plan de Supresión de Pasos a Nivel de RENFE ESPAÑA

RESUMEN

"No hay mejor paso a nivel que el paso muerto", suelen decir los encargados del tema. Pero, hasta hace muy poco, la eliminación de estos conflictivos cruces no sólo representaba mucho dinero, sino una larga obra, con la consabida secuela de precauciones, limitaciones de velocidad y problemas para el tráfico ferroviario. Sin embargo, un novedoso sistema, basado en clavar mediante gatos hidráulicos la estructura de hormigón del paso a diferente nivel en el terraplén de la vía, permite hacer en pocas semanas lo que antes se tardaba largos meses. La aplicación de este nuevo método reviste gran importancia,

especialmente si se piensa que las líneas a $200 \mathrm{~km} / \mathrm{h}$

prácticamente no pueden tener cruzamientos al mismo nivel.

\section{SUMMARY}

"The best level crossing is a dead crossing", those involved are accustomed to say. Yet, until a short time ago, eliminating these conflictive crossings not only implied a great deal of money, but prolonged building work, with the all too familiar sequel of precautions, speed restrictions and problems for rail traffic. However, a brand new system, based on using hydraulic jacks to drive the concrete structure of the crossing to a different level in the track embankment, allows the execution in a few weeks of what before took long months. Use of this new method acquires great importance, especially if one considers that $200 \mathrm{~km} / \mathrm{hour}$ tracks virtually cannot have crossings at the same level.

\section{INTRODUCCION}

El gran reto que se le plantea al ferrocarril español, de cara a la implantación de la alta velocidad, y necesidad de mantener el funcionamiento ininterrumpido de la comunicación ferrovaria incrementando su eficacia y mejorando los tiempos de viaje, hacen necesario disponer de técnicas que permitan la eliminación de los pasos a nivel en el menor tiempo posible y sin que esto conlleve un mayor coste.

En base a lo anterior de lo que se trata es, en cualquier caso, de buscar un "sistema sencillo pero no por ello fácil" de eliminar los pasos a nivel existentes, o los que se pudieran producir como consecuencia del desarrollo del actual sistema de carreteras o del ferrocarril.
En la actualidad no siempre y por razones técnicas, económicas y/o ambientales es posible la eliminación del paso a nivel mediante la construcción de un paso superior, entonces la solución es el paso inferior.

Lo que se ha dicho hasta ahora para la solución del cruce carretera-ferrocarril, como es obvio, puede ser extendido conceptualmente para cualquier otro sistema de infraestructura (canales, alcantarillas, servicios, etc.), en relación con la interferencia con el ferrocarril, incluso esta técnica que se expone a continuación podría servir para la traslación de pequeños edificios de carácter artístico, aunque en este artículo nos ceñiremos tan solo al caso de los pasos a nivel. 


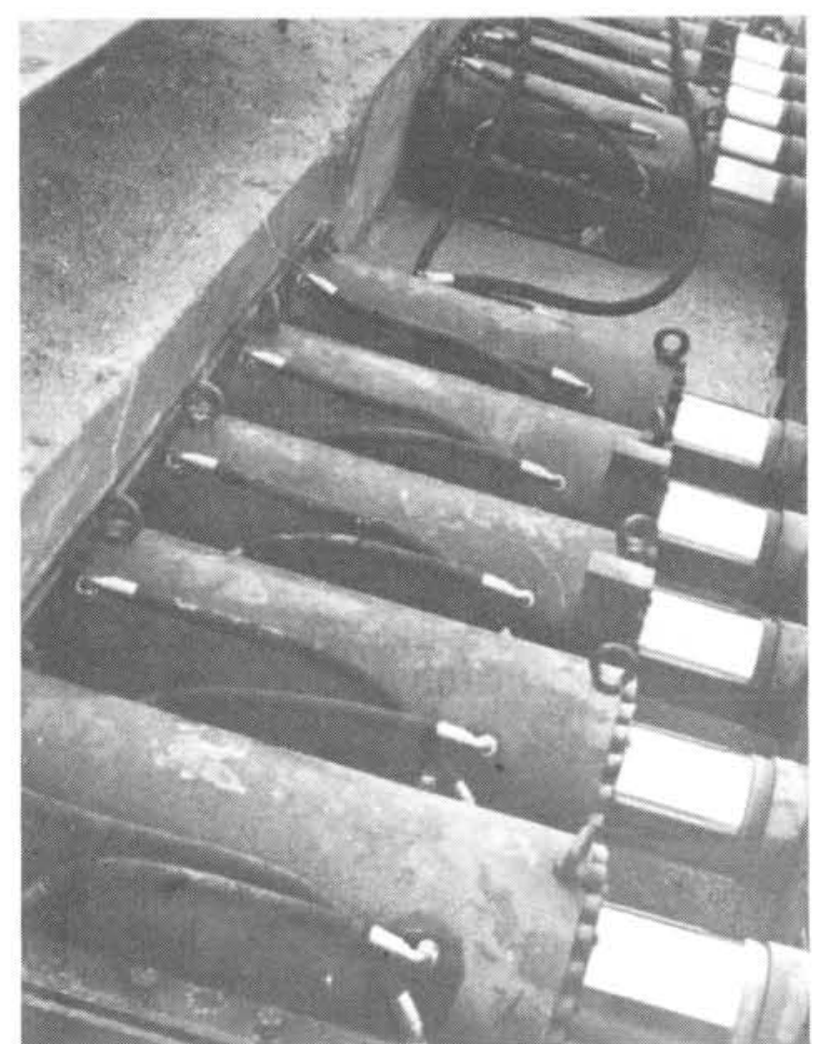

Fig. 1.-El cajón se empuja mediante una decena de gatos hidráulicos de gran tamaño.

En general, los estudios técnico-económicos sobre la construcción de un paso inferior bajo una linea de ferrocarril deben considerar, entre otras, las siguientes cuestiones:

- El alto coste de los retrasos que el tráfico del ferrocarril experimenta durante el tiempo requerido para la construcción de la estructura.

- El coste de la estructura que debe soportar temporalmente el tendido férreo, tanto durante el trabajo de excavación, como de forma definitiva.

- La necesidad de hacer compatible los niveles de servicio por ferrocarril, manteniendo los tiempos de entrega, con las necesarias operaciones de conservación de la via y de la duración de las obras de paso inferior.

- La seguridad de la obra en todas las fases de ejecución de la misma.

- El coste de pilotaje de este tipo de obras (personal del ferrocarril que se encarga de compatibilizar la necesaria permanencia del personal de obra en la via, con el tránsito normal de los trenes).

- Y por último, ya en el caso de RENFE, ante la puesta en marcha del plan de alta velocidad, la necesi- dad de realizar un elevado número de obras de supresión de pasos a nivel, minimizando las afeccio. nes a la circulación ferroviaria.

\section{CRITERIOS DE DISEÑO}

Cuando por diferentes razones (urbanísticas, topográficas, económicas, etc.), se decide que la solución más adecuada para la supresión de un paso a nivel es la realización de un paso inferior, el técnico se enfrenta no sólo al problema de diseñar la obra de fábrica, sino también a su puesta en obra, para lo que tendrá que tener en consideración las cuestiones de que se hablaba en el apartado anterior, máxime si el número de circulaciones ferroviarias es elevado.

Para resolver estas cuestiones se cuenta ahora, además de con todos los sistemas ya conocidos, con un nuevo método, conocido desde hace algunos años y ampliamente probado, consistente en la realización de la estructura fuera de su ubicación definitiva y su co. locación mediante traslación o empuje a su posición bajo las vías férreas.

El método tiene de original la técnica con que se ejecuta que, como ya se dijo anteriormente, consiste en colocar "empujando" bajo las vias, piezas prefabricadas que constituyen en si mismas pasos subterráneos para vehículos, peatones y conducción de servicios, sin más limitaciones que las que se exponen en el punto 4 de este artículo.

Los comentarios que siguen a continuación, pretenden exponer las peculiaridades y forma en que se ejecuta esta moderna metodología constructiva de modo esquemático y accesible para el máximo de personas interesadas en la realización de este tipo de trabajo.

\section{EL METODO DE EMPUJE}

Vamos a continuación a examinar las principales operaciones, tanto de fabricación como de colocación del cajón, refiriéndonos para ello a la obra realizada recien. temente mediante este método, para la supresión del paso a nivel en el P.K. 473/127 de la línea férrea MadridHendaya, en el Término Municipal de Lapuebla de Arganzón (Burgos).

\subsection{La solera y el muro de empuje (operaciones previas)}

Previo a la construcción del cajón o monolito se hace necesario crear el plano de asentamiento de éste, fue-

http://informesdelaconstruccion.revistas.csic.es 


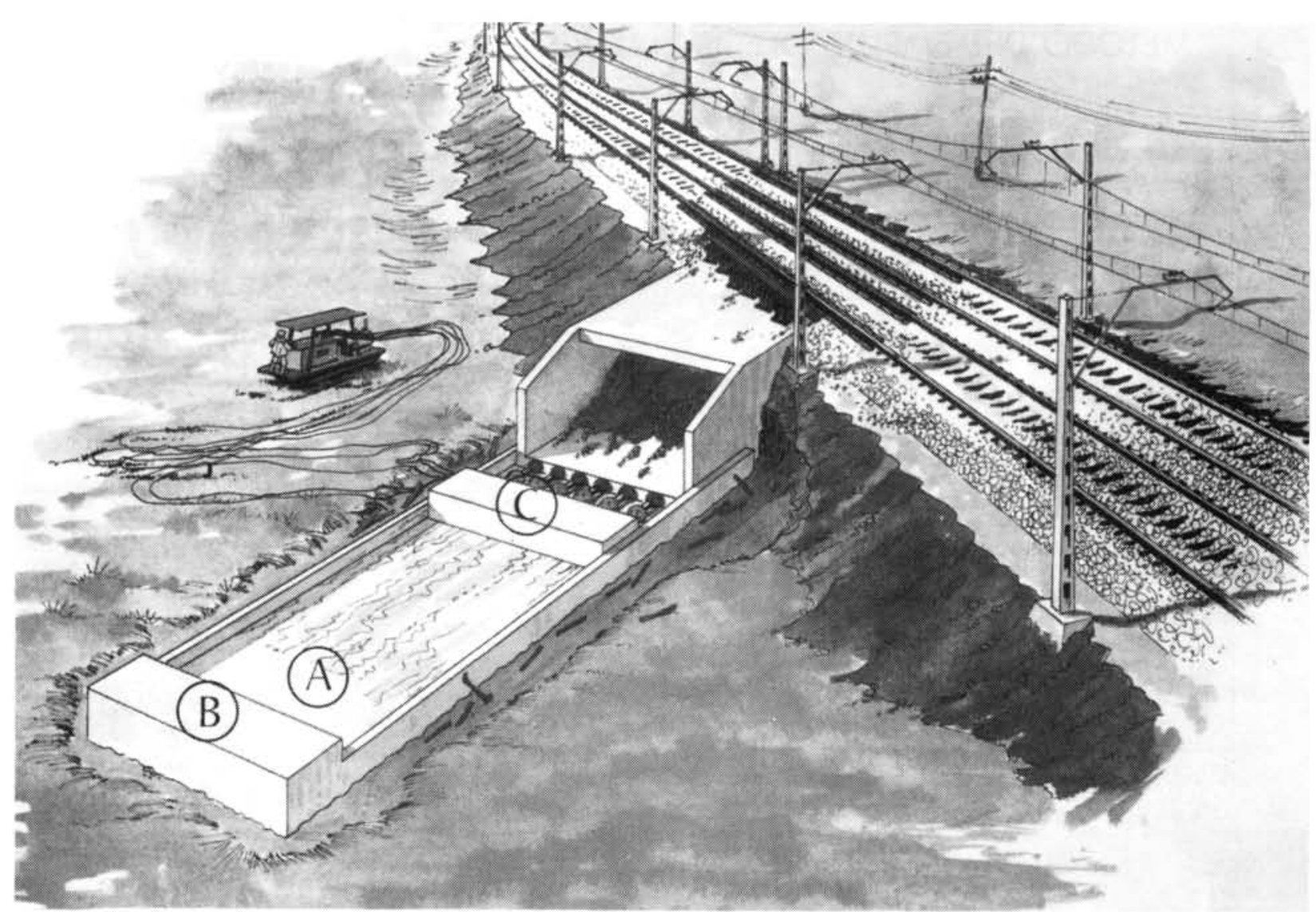

Fig. 2.-Procedimiento de hincado del cajón para obras de supresión de pasos a nivel.

ra de la obra, en principio distinto al definitivo, pero lo más próximo a él para reducir la distancia que el cajón debe recorrer y que, en cualquier caso, debe ser igual a la longitud de ésta más la necesaria para alojar el muro de empuje (el cual se describe a continuación), y los gatos de empuje.

Realizada la excavación anterior, por el procedimiento más adecuado en cada caso y que vendrá determinado por el terreno y espacio disponible, se procede a realizar la construcción de lo que denominaremos solera de deslizamiento (Fig. 2A) consistente, en este caso, en la construcción de una losa de $25 \mathrm{~cm}$ de espesor $y$ de $8,74 \mathrm{~m}$ de ancho y una longitud de $23,35 \mathrm{~m}$ de largo en hormigón armado, con unas guias laterales de $25 \mathrm{~cm}$, cuya función será evitar los desplazamientos laterales del cajón. La solera tiene una doble función: por un lado crear un plano de deslizamiento para el cajón y, por otro, servir de encofrado para la construcción del propio cajón.

En el extremo más alejado de las vias se ha construido el denominado muro de empuje de $90 \mathrm{~cm}$ de espesor y $3,00 \mathrm{~m}$ de alto, el cual está destinado a soportar el empuje de los diez gatos de $150 \mathrm{t}$ y que desarrollan en total 1.500 t para la obra en cuestión (Figs. 1 y 2B).
(En cada caso, tanto las dimensiones de la solera como del muro de empuje, y número de gatos, son función del peso del cajón a empujar).

Una vez construido el muro y la solera, y después del tiempo de curación del hormigón empleado, se extiende sobre éste una lámina de polietileno de cierto espesor, con una eventual capa de grasa lubricante, cuya función es además de separar la solera del cajón que se va a construir, la de reducir el rozamiento horizontal durante la traslación fundamentalmente en su fase inicial.

Durante la fase de empuje, cada 5 ó 10 metros, según los casos de avance, será necesario crear un segundo elemento (murete) de empuje fijo, antes de reemprender el empuje; este murete tiene como función reducir el número de elementos metálicos de apoyo de los que hablaremos a continuación y asegurar a su vez un nuevo muro de empuje y la correcta transmisión del esfuerzo.

Para la realizlación de la solera y muro de empuje se emplearon $90 \mathrm{~m}^{3}$ de hormigón de $250 \mathrm{~kg} / \mathrm{cm}^{2}$ de resistencia característica y $5.400 \mathrm{~kg}$ de acero de límite elástico $5.100 \mathrm{~kg} / \mathrm{cm}^{2}$ 


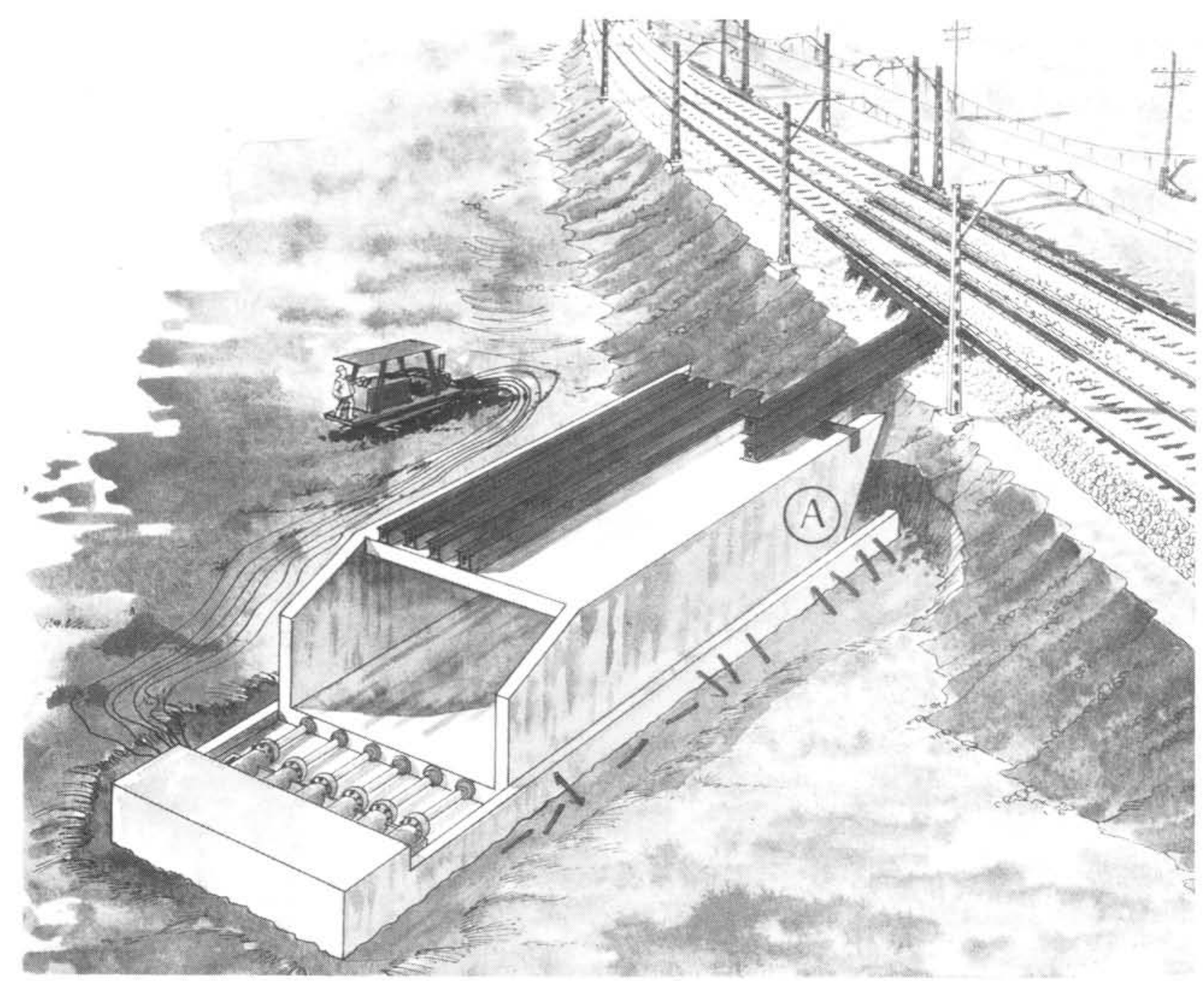

Fig. 3.-Supresión de un paso a nivel.

\subsection{El cajón}

El cajón construido y fuertemente armado, tiene en sus dos losas, usos distintos:

- La inferior soportará un camino de 7,00 $\mathrm{m}$ de ancho para el tráfico de vehículos carreteros.

- Y la superior, de 15,65 m, soportará el tráfico ferro. viario de una doble vía de alta velocidad.

En este caso, el cajón tiene una sección de $8,20 \mathrm{~m}$ por $5,30 \mathrm{~m}$, con un espesor constante de $0,60 \mathrm{~m}$ y una longitud en la losa inferior de 20,35 m.

El proceso de construcción usado para el encofrado es el de la técnica tradicional, con carpinteria de paneles de acero y apeos tubulares del mismo material.

Se han empleado $291 \mathrm{~m}^{3}$ de hormigón de $250 \mathrm{~kg} / \mathrm{cm}^{2}$ de resistencia caracteristica.
La cantidad de acero en este caso ha sido de $44.570 \mathrm{~kg}$ y de límite elástico $5.100 \mathrm{~kg} / \mathrm{cm}^{2}$; la cuantia de este último ha creado alguna dificultad en cuanto al vibrado que ha sido sumamente cuidado y laborioso.

En el proceso de cálculo se han tenido en cuenta no sólo las cargas recomendadas por la buena técnica, en cuanto al ferrocarril y la carretera se refiere, sino también la experiencia acumulada en otras actuaciones de este tipo.

Pasamos a continuación a comentar la curiosa forma del cajón y el porqué de la misma. En primer lugar es de destacar la forma de los estribos en su parte frontal (Fig. 3A), en forma de cuchillos, cuya finalidad es ofrecer la menor resistencia posible al avance contra el terreno y sujetar lateralmente el mismo, para lo que se biselan éstos en su plano de contacto con el terreno; en su parte superior, llevan un cajeo cuya función es alojar la viga de apoyo.

http://informesdelaconstruccion.revistas.csic.es 
Fig. 4.-Detalle de la viga que sirvió de apoyo a las de apeo transversal.
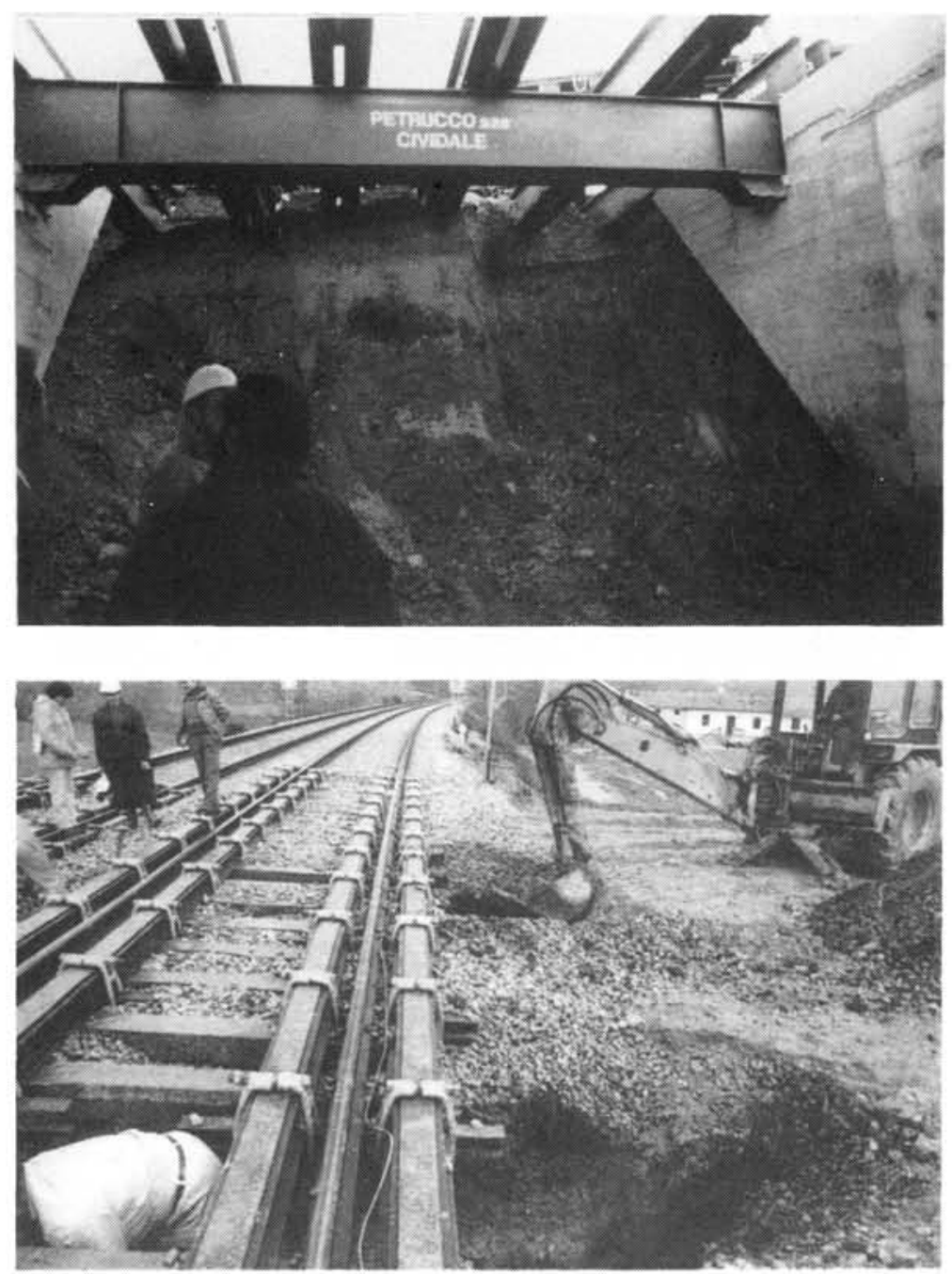

Fig. 5.-El avance de las vigas transversales, que abrian paso al cajón y sujetaban la vía transversal mente, iba precedido de la excavación del terreno afectado.

mo en este caso) o construida en hormigón según los casos, tiene como función servir de apoyo a los perfiles metálicos que han de soportar las vias y tiene en su parte superior soldados unos redondos que garantizan el deslizamiento del cajón bajo los perfiles me. tálicos, de los que hablaremos al tratar del apeo de vía.

\subsection{El apeo de via}

Una vez acabado el cajón y cuando éste ha alcanzado la resistencia caracteristica deseada, se procede al apeo de las vias, consistente en:

a) El apeo longitudinal, tradicional en este tipo de obras y consistente en colocar una pareja de paquetes de carriles paralelos a cada hilo de vía. En este caso los paquetes son de tres carriles sujetos por una brida (Fig. 5) a ambos lados del mismo y arriostrados por unos perfiles o carriles perpendiculares que hacen solidarios ambos paquetes entre sí, soportando, al tiempo, la carga de los carriles en el mo- 

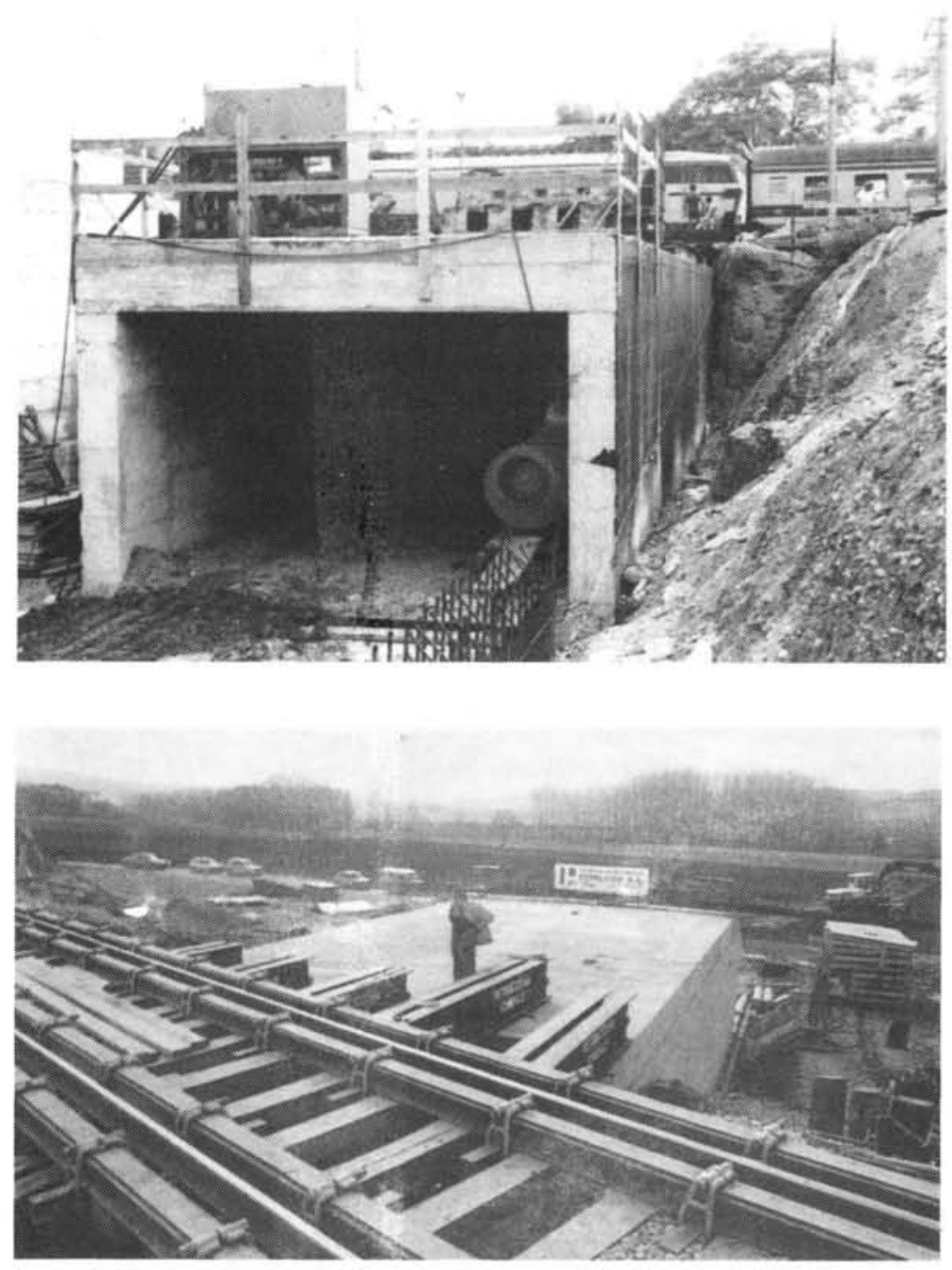

Fig. 6.-Vista de un cajón en proceso de colocación. Se puede observar el paso del tren sobre él.
Fig. 7.-Vista del cajón una vez comenzado el proceso de empuje. En primer término los apeos longitudinal y transversal, este último especifico para es. te sistema de construcción. mento en que, por necesidades de trabajo, desaparezca el apoyo del balasto. Este tipo de apeo presenta, en este caso, la peculiaridad respecto al sistema tradicional, de que la propia brida permite por su forma una sencilla y rápida colocación.

La longitud de estos apeos debe ser tal que no sólo permita la excavación necesaria para la colocación del cajón, sino también la posible aparición de unas facies de descompresión en el trasdós de los estribos del cajón y que según el tipo de terreno y el esviaje del cajón, respecto a las vías, puede alcanzar luces de 1 a 2 m (Fig. 6).

b) Y el apoyo transversal, característico de esta técnica constructiva, que se coloca bajo las traviesas y en la dirección del empuje y está compuesto por parejas empresilladas de IPN, en este caso de 600 (formando un perfil HEA), colocadas según el esviaje y separadas entre sí desde 1,20 a 2,40 m entre ejes. En el caso de la obra que sirve de referencia a este artículo, se colocaron 6 parejas de perfiles empresillados (Fig. 7).
La misión de este apoyo transversal es doble y sirve para sustentar la propia via y transmitir las cargas que ruedan sobre los carriles, repartiéndola sobre la losa del cajón y el propio terraplén y, en segundo lugar, crear un plano bajo el cual deslizar el propio cajón.

Los perfiles metálicos, cuya longitud es variable según el número de vías que componen el apoyo transversal, los cuales se suelen colocar bajo la traviesa, deben ser suplementados con cuñas de madera que sirven para garantizar el correcto nivel de la via en todo momento, y que son colocadas alternativamente para garantizar el correcto apoyo de las vias en todo momento.

Mención especial merece el caso en que es necesario el apeo de un cambio de vía, sustituyéndose las IPN por un emparrillado de perfiles que garantice en todo momento la correcta posición de éste y su perfecto funcionamiento.

Durante la fase de colocación, los perfiles se apo- 
yan sobre la losa del monolito y el terreno; este apoyo sobre el terraplén va desapareciendo progresivamente a medida que avanza el cajón, por efecto de la excavación, hasta que la carga de la vía se realiza sobre este último.

Para garantizar el deslizamiento cajón-IPN se colocan, entre éstos y el cajón, unos redondos que faciliten el mismo y se arriostran los perfiles en cabeza de empuje, de forma que no sufran movimiento con la continua traslación del cajón.

\subsection{El empuje}

Una vez realizadas las operaciones anteriores, se procede a iniciar la maniobra de empuje, momento en que conviene recordar a Manzonni cuando decía: "Súbito Pedro, pero con juicio", para lo que previamente se han colocado contra el alzado posterior de la solera inferior el juego de gatos (Fig. 1), en número suficiente para garantizar el esfuerzo necesario para mover el cajón, en este caso 10 gatos, interponiendo entre éstos y la solera una placa metálica que además de repartir la carga uniformemente, evita la aparición de fisuras en el cajón por efecto de las cargas puntuales que transmitirian los gatos.

Dentro del cajón y mediante unas rampas metálicas que se colocan al efecto, se procede a excavar un frente suficiente en el terreno (la excavación en su profundidad y forma es función del tipo de terreno) para per. mitir el avance del cajón en cortos recorridos, por lo general el correspondiente a la longitud del émbolo, aproximadamente $50 \mathrm{~cm}$.

La operación de traslación se realiza por razones de seguridad durante los intervalos en que no circulan trenes y una vez preparado el frente de avance.

Al final de cada avance de pistón de los gatos el espacio vacío que queda, entre el muro de empuje y el juego de gatos, es rellenado por unos elementos metálicos que tienen la misión de transmitir la carga de empuje en los sucesivos avances y que a partir de un determinado momento son sustituidos por cilindros metálicos, que se interponen entre los gatos y el muro.

Por lo general, al ser la traslación de varias decenas de metros, y por razones de seguridad, se hace necesario cada 5 ó 10 metros de avance la colocación de un murete que garantice la correcta transmisión del empuje (FIg. $2 \mathrm{C}$ ) y a la vez reduzca la necesidad de utilizar un elevado número de elementos metálicos de distanciamiento que disminuiría la seguridad del sistema. Es norma al uso realizar estos muretes aprovechando los cortes semanales de trabajo para no tener que detener el avance en las jornadas normales de trabajo.
Durante la operación de avance, puede ser necesario trasladar los perfiles transversales IPN que han permanecido inmóviles durante el empuje en la dirección de éste si su longitud no es suficiente para garantizar el correcto apoyo de todas las vías, para lo que se habrá preparado previamente el apoyo siguiente sobre el terreno y su traslación se realiza sin más maniobra que tirar de las mismas, de forma que la distancia entre apoyos de éstas sobre la viga apoyada en el cajeo de los estribos y el terreno permanezca por debajo de una cantidad determinada según el cálculo y tipo de IPN usada, arriostrándose los perfiles a continuación para evitar su traslación en sucesivos empujes.

De la descripción anterior se desprende fácilmente que los factores que, al fin y al cabo, determinan la velocidad de avance son la naturaleza del terreno y la organización de la excavación y retirada del mismo, asi como la coordinación entre estas excavaciones y el paso del ferrocarril.

La experiencia acumulada en la aplicación de esta técnica permite regular la velocidad de avance en recorridos de 1 a $3 \mathrm{~mm} / \mathrm{s}$ y conseguir traslaciones de 4 a $6 \mathrm{~m}$ en jornadas laborales de 8 horas. En el caso que sirve de ejemplo a este artículo, el empuje se empezó el lunes 30 de noviembre de 1988, dándose por concluido el viernes 4 de diciembre, con lo que la velocidad de avance fue de 5 metros/dia, si se tiene en cuenta que el miércoles 2 de diciembre se paró para realizar el murete de apoyo a que se aludió anteriormente.

La velocidad de avance en roca se reduce sensiblemente, aunque es fácil superar el metro/día, si la roca a excavar es susceptible de serlo con martillo, con lo que el avance sigue siendo mucho más rápido que cualquier otro método al uso.

Durante la obra y puesto que las fases de avance ocupan más de una jornada laboral, es buena norma, antes de la terminación de cada jornada de trabajo, tomar las debidas precauciones para evitar posibles movimientos del terreno y que suelen consister en:

- Hacer penetrar el cajón, suspendiendo la excavación con un empuje forzado para aguantar el terreno lateralmente con los cuchillos, y

- Abrigar el paramento de excavación con los materiales sueltos.

Por último, conviene recordar que pese a la sencillez del sistema, la facilidad de puesta en obra tan sólo se consigue cuidando en todo momento la excavación en su forma y cantidad, pues un error en ésta, ya sea por exceso o defecto, puede conducir a hundimientos o elevaciones del cajón que son difícilmente corregibles y que únicamente se consigue con intervenciones par- 

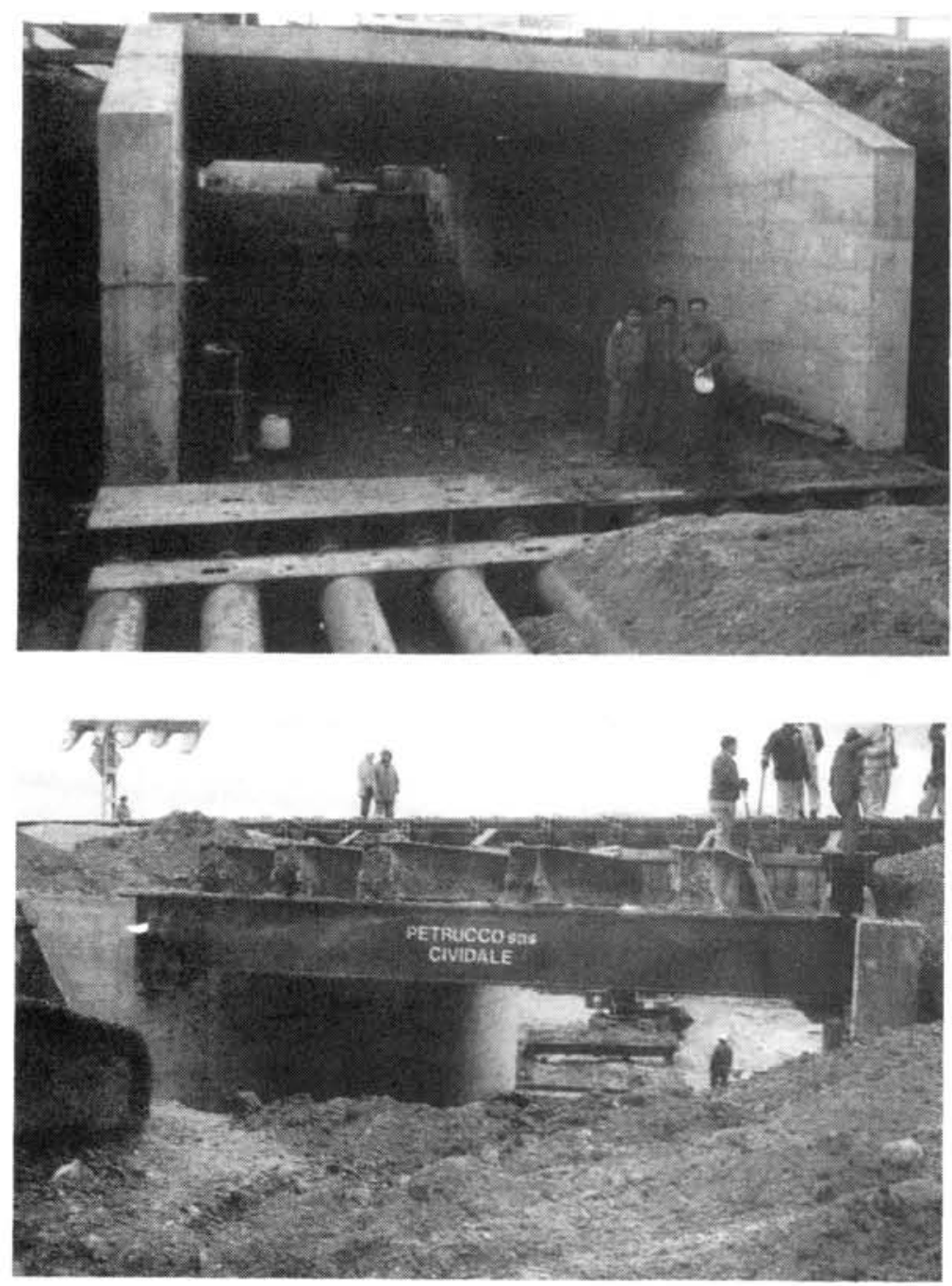

Fig. 8.-El cajón en su posición definitiva, con los gatos y las piezas metálicas (la prismática transver. sal y las cilindricas longitudinales) de empuje.

Fig. 9.-Acaba de terminar el empuje del cajón y so. lamente falta por retirar los apeos, restablecer el balasto $y$, por lo que respecta a la carretera, retirar las tierras del acceso.

ticularmente laboriosas y delicadas, que en cualquier caso ralentizan el proceso de avance y disminuyen la seguridad del procedimiento.

\subsection{Via libre}

Colocado ya el cajón en su posición definitiva (Fig. 8) bajo las vias, se procede a la retirada del apoyo transversal IPN en orden inversa al que se colocaron, sustituyendo el espacio vacio por balasto. En la obra que nos sirve de referencia y dada la diferencia de cotas entre la solera superior del cajón y la traviesa (doble via con curva), se ha considerado conveniente extender una primera capa de grava-cemento de unos $30 \mathrm{~cm}$ de espesor, para reducir la capa de balasto y que realizará las funciones de sub-balasto y a continuación se procede al extendido de balasto (Fig. 9).

Una vez extendido el balasto, se procede al bateo y nivelación de vía, según el método habitual para estos casos.
En total el tiempo en que se ha limitado la velocidad en este trayecto ferroviario ha sido de 12 dias a $30 \mathrm{~km} / \mathrm{h}$, más una semana con elevación escalonada de la mis. ma hasta conseguir la de trayecto. Durante esta semana se ha procedido a cambiar traviesas, controlando la nivelación de la vía.

\section{VENTAJAS Y LIMITACIONES DEL SISTEMA}

La rápida difusión que esperamos tenga el sistema de empuje en nuestro pais se justifica por sus ventajas intrínsecas. El cajón se ha construido a cielo abierto, con lo que no sólo se consiguen mejores calidades, sino también un mejor acabado e impermeabilización al realizarse ésta previa a su puesta en obra.

El rápido avance del cajón permite reducir al mínimo la afección a la circulación ferroviaria y elevar de 10 a $30 \mathrm{~km} / \mathrm{h}$ la precaución, con lo que se ha evitado la casi parada de los trenes. Esta ventaja se consigue al independizar la fase de fabricación de la estructura y la de su puesta en servicio. 
CUADRO 1

\begin{tabular}{|l|c|c|}
\hline \multirow{2}{*}{ UNIDADES DE OBRA } & \multicolumn{2}{c|}{$\begin{array}{c}\text { Tiempos en dias acumulados al origen } \\
\text { (Parciales) N. }{ }^{\circ} \text { de dias por U. de obra }\end{array}$} \\
\cline { 2 - 3 } & Sistema Pantallas & Sistema Empuje \\
\hline Cambio de traviesas & $2(2)$ & $2(2)$ \\
Apeo de vías & $5(3)$ & $5(3)$ \\
Excavación pantallas, encofrado y & $95(90)$ & - \\
hormigonado de estribos & $123(28)$ & - \\
Fraguado de hormigones & $125(2)$ & - \\
Montaje de losas & - & $\left({ }^{*}\right) 10(5)$ \\
Empuje cajón bajo via & - & $12(2)$ \\
Retirada perfiles, apeos y coloca- & $132(7)$ & $19(7)$ \\
ción balasto & 132 & 19 \\
Cambio de traviesas y nivelaciones & & 19 \\
\hline TOTAL TIEMPOS DE EJECUCION & &
\end{tabular}

(El cambio de traviesas es recomendable, pero no siempre necesario)

(*) Durante los cinco días de empuje se llevaron a cabo simultáneamente las operaciones de colocación de perfiles, diferentes desplazamientos de los mismos de acuerdo con el avance del cajón y hormigonado del murete para el avance del punto de empuje.

\section{CUADRO 2}

\begin{tabular}{|l|l|l|}
\hline \multicolumn{1}{|c|}{ PRECAUCIONES } & \multicolumn{1}{|c|}{ Sistema Pantallas } & \multicolumn{1}{c|}{ Sistema Empuje } \\
\hline Velocidad $\left(^{*}\right)$ & $\begin{array}{l}10 \mathrm{~km} / \mathrm{h} \text { en ambas vías } \\
\text { durante } 125 \text { dias }\end{array}$ & $\begin{array}{l}30 \mathrm{~km} / \mathrm{h} \text { en ambas vias } \\
\text { durante } 12 \text { días }\end{array}$ \\
\hline $\begin{array}{l}\text { Corte de via para mon- } \\
\text { taje de losas }\end{array}$ & $\begin{array}{l}\text { 2 días con duración de } \\
\text { 4 horas por día y vía }\end{array}$ & No necesario \\
\hline
\end{tabular}

(*) Durante la semana de cambio de traviesas y nivelaciones, la velocidad se va elevando escalonadamente hasta conseguir la velocidad habitual del trayecto.

CUADRO 3. CRONOGRAMA DE EMPUJE

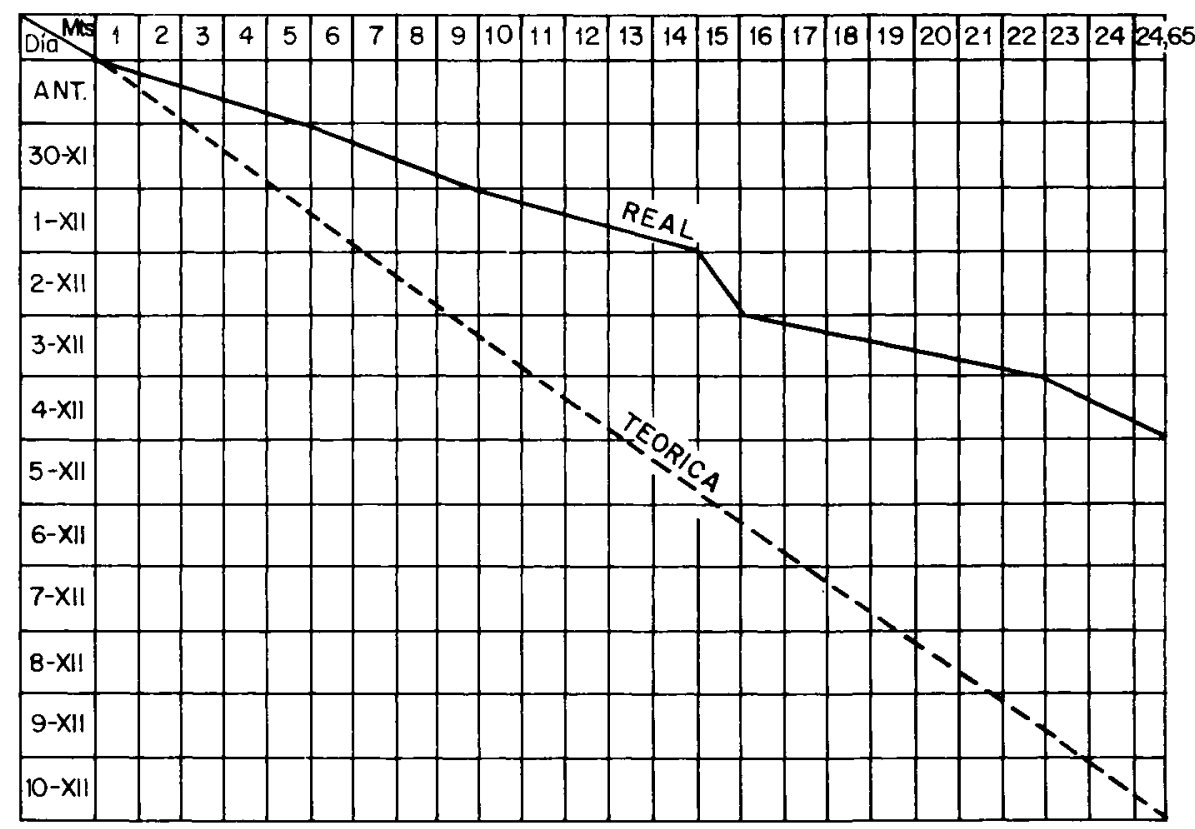


a) Día 25/11 - Avance: 3'00 Avance acumulado: 3'50
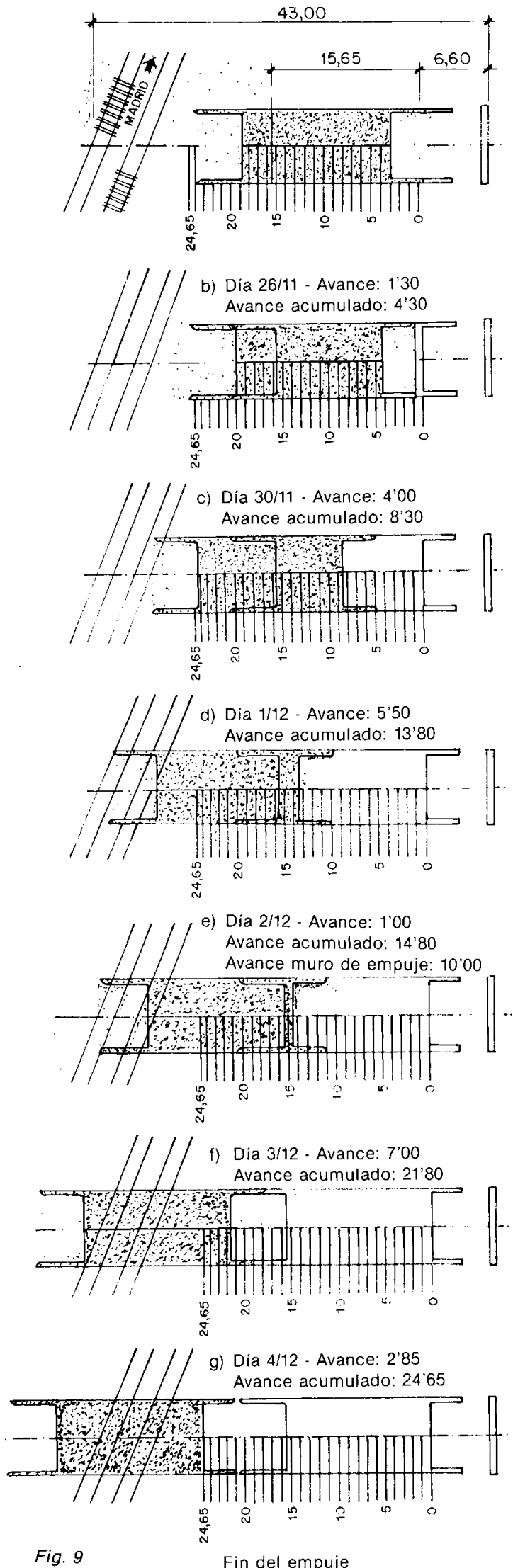

Fig. 9

Fases a) y b) - prueba de empuje y aproximación al terraplén.

Fases c), d), e), f) y g) - Empuje bajo vía.
Por lo que respecta al coste económico y referido únicamente a lo que es la estructura bajo vía cajón o su equivalente de pantallas fabricadas in situ y aplicado a la obra de Lapuebla de Arganzón, cabe decir que el coste absoluto, es decir considerando no sólo la ejecución material, sino también los costes de personal y retrasos de trenes como consecuencia de la duración de la precaución, es un $12 \%$ menor en el caso de utilizar el sistema de empuje que el tradicional de pantallas.

En cuanto a las limitaciones por el empleo de este sistema, que no creemos presente ningún inconveniente, la primera es la que pueda representar la capacidad portante del propio terreno que deberá ser superior a la carga que transmite el cajón y el propio ferrocarril.

La presencia de agua en las laderas o un nivel freático superior a la base de apoyo, que es otra fuerte limitación, está siendo estudiada y resuelta al aunar esta técnica con otras ya conocidas para la ejecución de este tipo de obras en presencia de agua.

La reducción de personal, tanto de vigilancia (pilotaje) como de obra y de maquinaria, unido al menor volumen en la mayoría de los casos de excavación y hormigones en la estructura, hacen que se vea compensado el mayor coste del empuje y del material utilizado en la solera y el muro e incluso la sobre-excavación que en algunos casos es necesario realizar.

A modo de resumen se incluyen los cuadros 1 y 2 , donde se compara este método con el tradicional de ejecución mediante pantallas.

Previo a la operación de empuje se llevó a cabo la ejecución del cajón, contrasolera y muro de empuje, con una duración, incluido fraguado, de 70 días. Teniendo en cuenta los tiempos totales de ejecución en el sistema de pantallas (132 dias) y de ejecución del cajón y empuje por este sistema $(19+70=89$ dias $)$, el ahorro conseguido para la marcha general de la obra es de 43 días.

Otra limitación, ésta meramente física, lo constituye la necesidad de disponer en la proximidad del punto de colocación del espacio necesario para fabricar el cajón, que en ocasiones tiene dimensiones muy considerables; estos espacios necesarios se ven a veces condicionados por la propia topografía del terreno (vertiente de montaña) que obliga a realizar una sobreexcavación y sobredimensionado del cajón en su gálibo vertical que luego deberá ser objeto de recrecidos en su losa inferior para adaptarse a la rasante definitiva del camino.

La presencia de edificación en la proximidad de la excavación requiere a veces la construcción de panta- 
Ilas que aseguren la estabilidad de éstos durante la excavación, hasta la cota de la solera de deslizamiento, pantallas que serán en cualquier caso utilizadas como muros de la obra definitiva.

En ocasiones y cuando la limitación de espacio lo exige, o la longitud del cajón lo requiere (la longitud probada como éxito máximo es del orden de 30 ó 40 metros), se recurre a realizar elementos-cajón más cortos que se van suplementando, empezando siempre por colocar el estrictamente necesario para la sustentación de la via.

En cualquier caso es necesario, y como operación previa, proceder al desvío o retirada de cuantas conducciones o servicios ferroviarios o urbanos puedan entorpecer el normal desarrollo del empuje.

Como conclusión, podemos decir que la técnica descrita, netamente ferroviaria, está concebida para ofre- cer una solución al problema del mantenimiento del tránsito ferroviario y la necesidad de realizar un número importante de obras de supresión de pasos a nivel o de paso bajo éste, de servicios de gran dimensión, problema que se viene presentando en los últimos años de forma creciente.

\section{ULTIMAS REALIZACIONES}

Recientemente, y con éxito, se ha llevado a cabo una nueva experiencia con este sistema, consistente en la colocación de un cajón en roca -en el Ayuntamiento de Arrigorriaga (Vizcaya) - convinando la Técnica ya descrita con la voladura controlada, donde se alcanzaron avances superiores a 1 metro/día, con lo que se demuestra que el sistema prácticamente no tiene limitaciones al haberse resuelto ya los problemas derivados de la presencia de roca y agua.

\section{publicaciones del ICCET/CSIC}

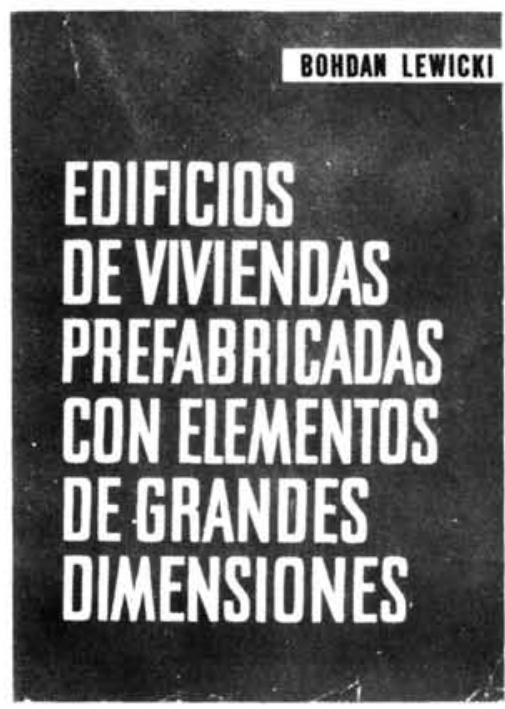

Bohdan Lewicki

Este libro trata de los problemas relativos a la construcción de los edificios de viviendas - publicos realizados con elementos prefabricados de grandes dimensiones. Se han estudiado los problemas de arriostramiento, asi como los que plantea la resistencia de los elementos y de la estructura; se han examinado las cuestiones de orden higrotermico, acústico y de resistencia al fuego: también se ha profundizado en el estudio de la estanquidad de los muros exteriores y de las juntas.

La obra incluye numerosas ilustraciones que dan detalles de diversas soluciones, as como ejemplos de cálculo, tabias de valores numéricos, diagramas y ábacos.

Un volumen encuadernado en tela, de $24 \times 17 \mathrm{~cm}$, compuesto de 616 págs.

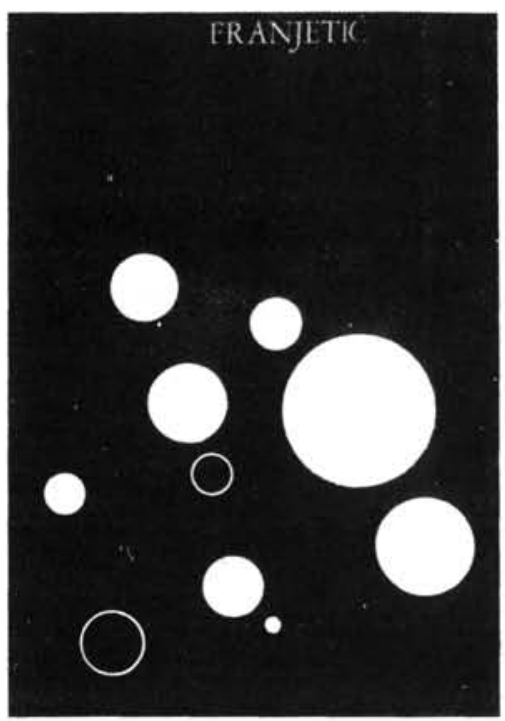

Zorislav Franjetic

En la obra de Franjetic se expone de una forma minuciosa, ordenada y sistemática, todo un cuerpo de doctrina que reúne el conocimiento actual sobre el endurecimiento rápido del hormigón. Parte el autor de los principios básicos y llega a las últimas consecuencias y realidades técnicas y econo micas.

Es una obra de consulta, tanto para el investigador sobre la materia, como para el proyectista y el realizador y montador de plantas e instalaciones y equipos de curado y endurecimiento rápido.

Un volumen encuadernado en cartóne, de $17 \times 24,5 \mathrm{~cm}$, compuesto de 385 págs. 110 figuras y 10 tablas.

Precios: 2.500 ptas.; \$USA 36.00 .

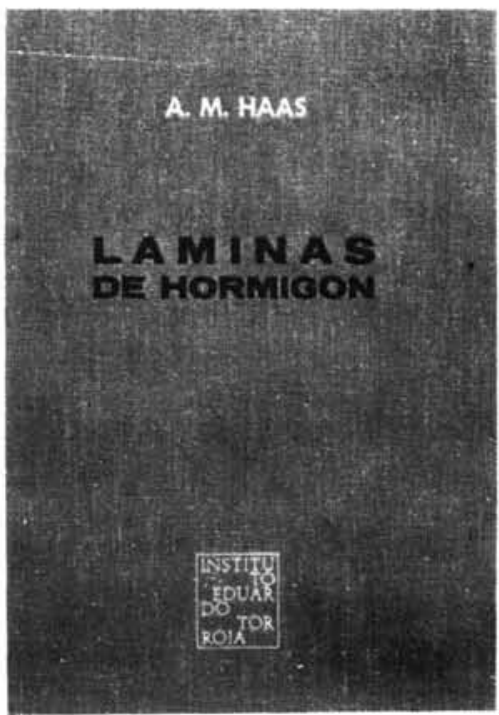

A. M. Haas

Al escribir este libro el autor intento poner a disposición de los estudiantes y de los ingenieros unos conocimientos prácticos, adecuados para servir de guia en el diseño y construcción de láminas delgadas de hormigón.

El autor está convencido de que el éxito en el diseño de una lámina exige, por parte del proyectista, un examen de las tres fases por las que pasa la materialización de la lámina: el diseño, el análisis estructural y la cons. trucción de la estructura.

Un volumen encuadernado en tela, de $17 \times 24,5 \mathrm{~cm}$, compuesto de 420 oágs., 141 figuras, 22 fotografias y 6 tablas.

Precios: 2.500 ptas.: SUSA 36.00

Precios: 2.500 ptas.; SUSA 36.00 . 